\title{
Characterization of the humoral immune response to glutamic acid decarboxylase in patients with autoimmune polyendocrinopathy-candidiasis-ectodermal dystrophy (APECED) and/or type 1 diabetes
}

\author{
Matti S Ronkainen, Taina Härkönen ${ }^{1}$, Jaakko Perheentupa ${ }^{1}$ and Mikael Knip ${ }^{1,2}$ \\ Department of Pediatrics, University of Oulu, PO Box 5000, FI-90014 University of Oulu, Oulu, Finland, ${ }^{1}$ The Hospital for Children and Adolescents, \\ University of Helsinki, PO Box 281, FI-OOO29 HUCH, Helsinki, Finland and ${ }^{2}$ Department of Pediatrics, Tampere University Hospital, PO Box 2000 , \\ FI-33521 Tampere, Finland
}

(Correspondence should be addressed to M Knip at the Hospital for Children and Adolescents, University of Helsinki, PO Box 281, FI-OOO29 HUCH, Helsinki, Finland; Email: mikael.knip@hus.fi)

\begin{abstract}
Objective: A humoral autoimmune response to glutamic acid decarboxylase (GAD65) is common both in patients with type 1 diabetes and in those with the autoimmune polyendocrinopathy-candidiasisectodermal dystrophy (APECED) syndrome, while overt type 1 diabetes is relatively rarely diagnosed in APECED patients. The aim of this study was to assess whether this difference in the incidence of type 1 diabetes is associated with variability in the humoral immune response to GAD65, one of the major autoantigens in type 1 diabetes.

Methods: Epitope- and isotype-specific GAD65 autoantibodies were analysed in 20 patients with APECED and 20 patients with newly diagnosed type 1 diabetes alone by radiobinding assays.

Results: GAD65 autoantibodies targeted the middle and carboxy-terminal regions of GAD65 and occasionally the amino-terminal region in the APECED patients and comprised mainly the IgG1 subclass and less frequently the IgG2 and IgG4 subclasses. The profile of epitope- and isotype-specific GAD65 autoantibodies was similar in type 1 diabetes and APECED, except that IgG2 subclass antibodies were observed more often and at higher levels in the patients with type 1 diabetes alone $(P<0.05)$. None of the measured parameters separated APECED patients with type 1 diabetes from those without type 1 diabetes.

Conclusion: APECED-associated humoral autoimmunity to GAD65 does not differ markedly from that observed in type 1 diabetes; only IgG2-GAD65 antibodies may be more closely associated with the latter entity.
\end{abstract}

European Journal of Endocrinology 153 901-906

\section{Introduction}

Autoantibodies to the gamma-aminobutyric acid (GABA) synthesizing glutamate decarboxylase (GAD) occur frequently in type 1 diabetes, polyendocrine autoimmune diseases and some neurological diseases $(1-4)$. Two isoforms of GAD are expressed in man, with molecular masses of 65000 and $67000 \mathrm{Da}(5$, 6). Antibodies to GAD65 (GAD65Abs) occur at diagnosis in up to $80 \%$ of patients with type 1 diabetes, whereas antibodies to GAD67 (GAD67Abs) are detected infrequently and probably as a result of epitopes shared with GAD65 (7). Autoantibodies to GAD65 are also common in patients with autoimmune polyendocrinopathy-candidiasis-ectodermal dystrophy (APECED, also known as autoimmune polyendocrine syndrome type 1), a rare autosomal recessive disease linked to mutations in the AIRE gene on chromosome $21(2,8-10)$. Type 1 diabetes is a component of APECED in up to $23 \%$ of patients (11). Since GAD65Abs occur in some $40 \%$ of APECED patients, however, their positive predictive value for future type 1 diabetes is low in these cases $(8,12)$. This is in contrast to persons with preclinical isolated type 1 diabetes, whose GAD65Abs are closely associated with progression to diabetes (13). Even so, some of these susceptible individuals fail to progress, as is also the case with the majority of GAD65Ab-positive patients with APECED. Such GAD autoimmunity may, at least partly, represent non-destructive islet cell autoimmunity. Autoantibodies to GAD65 target mainly conformational antigenic determinants located in the middle 
and C-terminal regions of GAD65 (14-17) and are composed of the IgG1 subclass and to a lesser extent other IgG subclasses in cases of type 1 diabetes (18). In patients with APECED, GAD65Abs are present at higher levels (8), more frequently share epitopes with GAD67 (8), and may more often recognize linear epitopes and be reactive to epitopes other than those observed in classical type 1 diabetes (19). Since there is limited information available on the detailed characteristics of humoral autoimmunity to $\mathrm{GAD}$ in patients with APECED, we set out to characterize reactivity to three major epitope clusters of GAD65 and the isotype distribution within the humoral response to GAD65 in a series of GAD65Ab-positive patients with APECED, comparing them with patients with newly diagnosed type 1 diabetes alone.

\section{Subjects and methods}

\section{Patients}

The subjects were derived from a series of patients with APECED diagnosed in Finland up to 1988 (10). The patients whose sera were available and who had been observed to be GAD65Ab-positive were retested in our laboratory in Oulu, and 20 of them (13 male, 65\%) were confirmed positive. They all had chronic mucocutaneous candidiasis together with hypoparathyroidism and/or primary adrenocortical failure. Altogether, thirteen of them $(65 \%)$ had hypoparathyroidism, seven (35\%) adrenocortical failure and six (30\%) clinical type 1 diabetes. Their mean age at the first sample with detectable GAD65Abs was 22.4 years $( \pm 11.4$ (S.D.), range 4.3-47.9). Serum samples were available, 1-8 for each patient, taken at irregular intervals and stored at $-20^{\circ} \mathrm{C}$. Two patients had one sample with detectable GAD65Abs, four had two samples, two had three samples, five had four samples, three had five samples, two had seven samples, and two had eight samples. The median duration of the GAD65Ab-positive period was 10.4 years (range 1.1-23.8) in those with more than one sample positive for GAD65Abs. The six cases of clinical type 1 diabetes had been diagnosed at age $19.3,19.6,23.5,35.9,43.3$ and 45.3 years, the individual sample closest to the diagnosis having been obtained at age 34.1, 19.2, 23.5, 36.2, 42.3 and 42.7 years respectively. The patients with type 1 diabetes alone were selected from a series of 352 newly diagnosed patients admitted to the Departments of Paediatrics and Internal Medicine, Oulu University Hospital, in 1988-1995 (20). These were matched with the APECED patients for age (mean \pm S.D., $21.4 \pm 11.6$ vs $22.4 \pm 11.4$ ), gender (13 male and 7 female) and levels of total GAD65Abs (median 100.1 vs 132.1 relative units (RU)). One inhibition analysis was not performed in one patient with type 1 diabetes alone due to insufficient serum volume, and this APECED-type 1 diabetes pair was excluded from the statistical analysis. Two subjects with type 1 diabetes alone were re-matched with each of the six diabetic APECED patients based on their age at the sample closest to the diagnosis of diabetes (mean \pm S.D., $24.8 \pm 11.6$ vs $32.1 \pm 10.9$; median levels of GAD65Abs, 115.8 vs $87.2 \mathrm{RU})$. When the APECED patients with diabetes were compared with those without, the first available GAD65Ab-positive sample was used. All the APECED patients had been examined at the Hospital for Children and Adolescents in Helsinki, and the study was approved by the local Ethical Committees. The patients gave their written informed consent.

\section{Methods}

Antibodies to human full-length GAD65 and its three major epitope clusters and to human full-length GAD67 were determined with a radiobinding assay using ${ }^{35}$ S-labelled full-length proteins or GAD65/67 chimaeric molecules $(21,22)$. Radiolabelled antigens were produced in an in vitro transcription/translation system (Promega, Madison, WI, USA). The chimaeric molecules used were GAD65 $1-95 / \mathrm{GAD} 67_{102-593}$ for N-terminal GAD65 epitopes, GAD67 $1-101 /$ GAD65 $5_{96}$ ${ }_{444} / \mathrm{GAD} 67_{453-593}$ for epitopes in the middle region of GAD65 and GAD67 1 -453/GAD65 ${ }_{445-585}$ for C-terminal GAD65 epitopes (17). cDNAs of the chimaeric constructs were gifts from Ezio Bonifacio (Milan, Italy). The conformations of these chimaeric proteins have been validated using monoclonal antibodies and competitive inhibition tests (17), and that of the GAD65 molecule by monoclonal antibodies recognizing conformational epitopes in the middle domain (MICA4) and the C-terminal domain (MICA3) of GAD65. The levels of antibodies were expressed as RU based on a standard curve run on each plate. The cut-off limit for antibody positivity was set to represent the 99th percentile in 373 non-diabetic Finnish children and adolescents in the GAD65 Ab assay or 104 subjects in the epitopespecific GAD65Ab and GAD67Ab assays. These limits were $5.35 \mathrm{RU}$ for GAD65Abs, 0.86 RU for N-terminal antibodies (GAD65-N-Abs), 1.51 RU for middle region antibodies (GAD65-M-Abs), 1.59 RU for C-terminal antibodies (GAD65-C-Abs) and 0.91 RU for GAD67Abs. Since the levels of GAD67Abs were high in many samples, inhibition experiments were performed to confirm specific binding. All initially GAD65Ab-positive samples were re-analysed for specific GAD65Abs in an inhibition assay with unlabelled GAD67, all initially GAD67Ab-positive samples for specific GAD67Abs were re-analysed in an inhibition assay with unlabelled GAD65, and all samples initially positive for GAD65-N-Abs, GAD65M-Abs and/or GAD65-C-Abs for specific GAD65 epitope binding were re-analysed in inhibition assays with unlabelled GAD67. The unlabelled antigens were produced by the same method as the labelled antigens but in the absence of ${ }^{35} \mathrm{~S}$-methionine. The serum 
samples were diluted for the inhibition assays to provide an uninhibited binding of approximately 1000 c.p.m. If the binding was inhibited by $80 \%$ or more upon adding the unlabelled antigen, the sample was considered to lack specific binding for the labelled antigen. In all assays, low and high standard serum samples were included on each plate to monitor the precision of the assay. The coefficients of interassay and intra-assay variation in our assays were maximally $25 \%$. The disease sensitivity of the GAD65Ab assay was $82 \%$ and the specificity $98 \%$ in the 2002 Diabetes Antibody Standardization Programme (DASP) workshop. Isotype-specific GAD65Abs were analysed with a methodology similar to that used for the assay of full-length GAD65Ab except that the protein A Sepharose precipitation was replaced by monoclonal anti-human Ig antibodies linked to streptavidin agarose. Briefly, the streptavidin agarose beads were washed thoroughly with buffer before use, and the biotinylated monoclonal anti-human IgG1, IgG2, IgG3, IgG4, IgA, IgE and IgM antibodies were linked to them by joint incubation. The beads were then washed with buffer. Finally, they were suspended in buffer and used for the precipitation. A more detailed procedure has been published recently (22). The results were expressed as S.D. scores (SDS) calculated as: SDS $=$ [delta c.p.m. $(=$ IgG subclass or isotype-specific c.p.m.-unspecific anti-rat IgM c.p.m.) - mean delta c.p.m. of control subjects]/s.D. delta c.p.m. of control samples as described previously (18). Forty non-diabetic young Finnish subjects (mean age $9.9 \pm 4.0$ (S.D.), range $0.6-16.6$ years) served as controls. The threshold for positivity was set at 3 SDS. Matched sample pairs were run as far as possible in the same isotype-specific GAD65Ab assays to eliminate the effect of interassay variation. Samples were reanalysed if the coefficient of variation in parallel samples exceeded 20\%. A series of other samples were re-analysed for quality control purposes. We participated in the serum exchange workshop carried out between the laboratories known to measure isotype responses to GAD65 (results presented at the 5th International Congress of the Immunology Diabetes Society 2001, Chennai, India). Our results were highly concordant with those of other laboratories using a similar method.

\section{Statistical analysis}

Chi-square statistics and Fisher's exact test were used as appropriate to evaluate differences in the frequencies of antibodies, and the Mann-Whitney U-test was used to compare antibody levels between the patient groups. The statistical analyses were performed using the SPSS statistical software package, version 10 (SPSS, Chicago, IL, USA) or Arcus QuickStat Biomedical Statistical Software (Addison Wesley Longman, Research Solutions, Cambridge, Cambs, UK).

\section{Results}

The 20 APECED patients previously documented as having tested positive for GAD65Abs were confirmed to be positive in our laboratory in Oulu. Sixteen of them were positive from the first available sample to the last follow-up sample, three seroconverted to stable GAD65 positivity during observation, and one was positive for GAD65Abs in the first two samples, seroconverted to negativity and then re-converted to positivity later. Antibodies to GAD67 were observed in 16 of the 20 patients during follow-up. Antibodies to the GAD65 ${ }_{1-95} /$ GAD67 $102-593$ molecule were detectable in 15 of the 20 APECED patients, in nine of whom specific binding to the N-terminus of GAD65 persisted after GAD67 inhibition. One patient presented with a highly N-terminal-specific response, since GAD67 was not capable of inhibiting the binding of GAD65 $1-95 /$ GAD67 $102-593$ at all in any of her five samples. Antibodies to the middle GAD65 construct, GAD67 $7_{1-101} / \mathrm{GAD} 65_{96-444} / \mathrm{GAD} 67_{453-593}$, were seen in 19 of the 20 APECED patients. GAD67 did not inhibit the binding totally in any of the samples, confirming the presence of specific GAD65-M-Abs. Similarly, specific Cterminal GAD65Abs were observed in all the samples that showed binding to the GAD67 $1-453 /$ GAD65445585 molecule. Altogether, 18 of the 20 APECED patients had GAD65-C-Abs. When an epitope-specific response appeared, it tended to remain: GAD65-M-Abs and GAD65-C-Abs disappeared along with the disappearance of total GAD65Abs and re-appeared later in one patient, while the disappearance of the specific C-terminal and N-terminal responses was seen in one patient. IgG1-GAD65Abs were detected in 19 of the 20 patients and their levels were higher than those of any other IgG subclass or isotype. If GAD65Abs were present, an IgG1 response was detectable except in one sample with low levels of total GAD65Abs. IgG4-GAD65Abs were observed in 17 patients and IgG2-GAD65Abs in 15, whereas IgG3-GAD65Abs were detectable in only two patients. An IgG4 response was present or appeared as a continuous response in eleven patients, fluctuated in four and seroconverted to permanent negativity in two initially positive patients. IgG2 responses were more variable, a continuous response or seroconversion to permanent positivity occurring in five patients, fluctuating or single responses in five and inverse seroconversions in five. Both of the IgG3 responses detected were present in the first samples included and disappeared thereafter. There were no differences in the distribution of epitope-specific and isotype-specific GAD65Abs between the patients with APECED and those with type 1 diabetes alone except for the higher prevalence and levels of Ig2-GAD65Abs in the latter $(P<0.05$, Fig. 1 , Table 1$)$. The finding of higher titres of IgE class antibodies in patients with type 1 diabetes alone is controversial, since the levels of IgEGAD65Abs were in most cases below the cut-off limit 


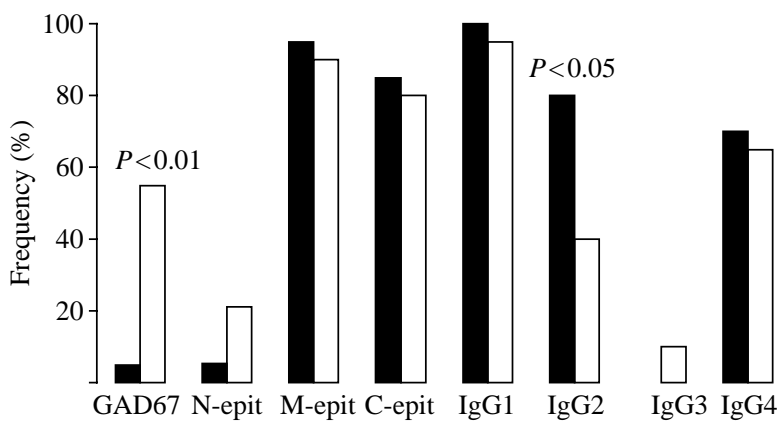

Figure 1 Frequencies of GAD67Abs and epitope- and IgG subclass-specific GAD65Abs in patients with type 1 diabetes $(n=20$, solid bars) and APECED ( $n=20$, open bars). The differences in antibody frequencies were evaluated with Fisher's exact test. The difference in the frequency of GAD67Abs was 0.50 (95\% confidence interval $(\mathrm{Cl}) 0.23-0.71)$ and the difference in the frequency of IgG2 was $0.40(\mathrm{Cl} 0.09-0.64)$. N-epit, N-terminal antibodies ( $n=19$ in both groups, since sufficient serum volume was unavailable from one patient with type 1 diabetes); M-epit, middle region antibodies; C-epit, C-terminal antibodies.

for antibody positivity. Antibodies to GAD67 were more common and showed higher levels in the patients with APECED than in those with type 1 diabetes alone $(P<0.01$, Fig. 1, Table 1). The distribution of epitopeand isotype-specific antibodies did not differentiate APECED patients with type 1 diabetes from non-diabetic patients or from those with type 1 diabetes alone (Figs 2 and 3). No apparent changes occurred in the epitope or isotype profile at the time of diagnosis of type 1 diabetes in the patients with APECED. Antibodies to GAD67 were more frequent $(P<0.05)$ and present at higher levels $(P<0.01)$ in the APECED patients with type 1 diabetes than in the patients with type 1 diabetes alone (Fig. 2). Antibodies to GAD67 were observed in the APECED patients irrespective of whether type 1 diabetes was present (Fig. 3).

\section{Discussion}

The aim of this study was to characterize the humoral immune response to GAD in APECED patients in detail

Table 1 median levels (RU or SDS) of antibodies in patients with type 1 diabetes $(n=20)$ and APECED $(n=20)$.

\begin{tabular}{lccc}
\hline & Type 1 diabetes & APECED & $\begin{array}{c}\boldsymbol{P} \text {-value } \\
\text { (Mann-Whitney U) }\end{array}$ \\
\hline GAD65 & 100.1 & 132.1 & 1.000 \\
GAD67 & 0.2 & 2.4 & $0.002^{\star}$ \\
IgG1-GAD65 & 338.6 & 681.2 & 0.10 \\
IgG2-GAD65 & 7.3 & 1.7 & $0.024^{\star}$ \\
IgG3-GAD65 & 0.1 & 0.1 & 0.43 \\
IgG4-GAD65 & 6.2 & 5.3 & 0.84 \\
IgA-GAD65 & 4.0 & 5.7 & 0.23 \\
IgE-GAD65 & 1.0 & 0.1 & $0.017^{\star}$ \\
IgM-GAD65 & 7.8 & 13.3 & 0.16 \\
\hline
\end{tabular}

* $P$ values are significant.

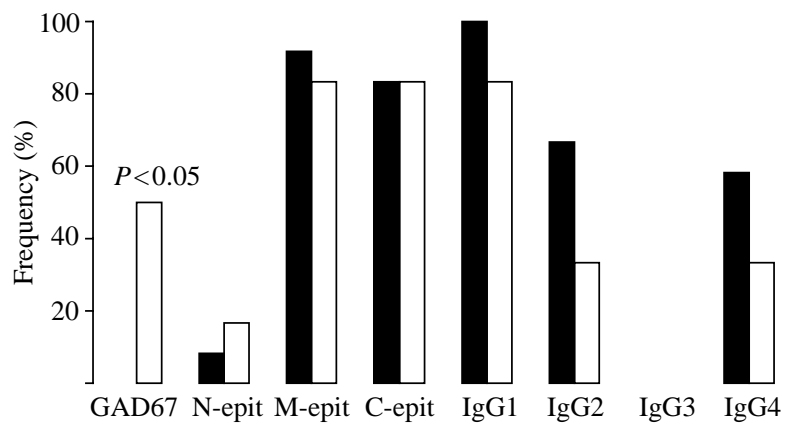

Figure 2 Frequencies of GAD67Abs and epitope- and IgG subclass-specific GAD65Abs in patients with type 1 diabetes alone ( $n=12$, solid bars) and APECED with type 1 diabetes $(n=6$, open bars). The differences in antibody frequencies were evaluated with Fisher's exact test. The difference in the frequency of GAD67Abs was 0.50 (Cl 0.17-0.82). N-epit, N-terminal antibodies; M-epit, middle region antibodies; C-epit, C-terminal antibodies.

by analysing antibodies to three major epitope clusters and isotype-specific responses. We observed that the humoral immune response to GAD mainly targets the middle and C-terminal regions of the GAD65 molecule, while a response to the N-terminus was seen in about half of the patients. The response comprised IgG1 subclass antibodies in almost all patients, whereas IgG2 and IgG4 subclass antibodies occurred in $60-80 \%$ of them. We also confirmed previous reports that antibodies to GAD67 cross-reacting with GAD65 are frequent in patients with APECED $(8,19)$. In the present study, the APECED patients were matched with type 1 diabetic patients according to, among other things, total GAD antibody levels. The same criterion was applied when matching APECED patients affected by type 1 diabetes to those unaffected. The matching was relatively crude, as it was based on GAD antibody levels obtained without diluting strongly positive samples. The crude matching might potentially result in false positive differences due to unrevealed differences in total GAD antibody levels between the

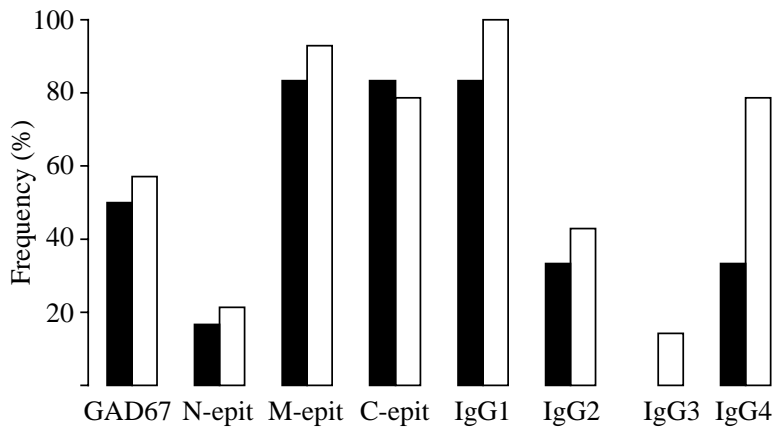

Figure 3 Frequencies of GAD67Abs and epitope- and IgG subclass-specific GAD65Abs in APECED patients with type 1 diabetes ( $n=6$, solid bars) and without type 1 diabetes $(n=14$, open bars). $\mathrm{N}$-epit, $\mathrm{N}$-terminal antibodies; $\mathrm{M}$-epit, middle region antibodies; C-epit, C-terminal antibodies. 
groups. The major finding of this study is, however, that the humoral immune response to GAD was surprisingly similar in patients with APECED and/or type 1 diabetes. No detailed epitope- and isotype-specific information has been available on the humoral immune response to GAD in APECED patients. It has been stated that mainly conformational epitopes located in the middle and C-terminal region of GAD65 are immunogenic in type 1 diabetes (16), and we similarly observed a strong response to both of these regions in APECED patients. We also detected epitope spreading from the middle region to the C-terminus in two patients, again an observation that is consistent with those observed in patients with type 1 diabetes $(14,15$, 17). Sera from APECED patients have been observed to bind denatured GAD65 and to inhibit the enzymatic activity of GAD65 but not sera from patients with type 1 diabetes alone (19). This may be reflected by different patterns of epitope recognition. In several patients with APECED we observed reactivity to the N-terminus that was not inhibited completely by GAD67. Similarly, GAD65Abs recognize linear epitopes in the N-terminus of the molecule in the stiff-man syndrome, a rare neurological disease characterized by exceptionally high titres of GAD65 Abs and the presence of type 1 diabetes in one-third of GAD65Ab-positive patients (23, 24). However, we observed no statistical difference in the frequency of N-terminal responses between the matched series of patients with APECED and type 1 diabetes alone.

To summarize, the humoral immune response to the three major epitope clusters of GAD65 is surprisingly similar in patients with APECED and patients with type 1 diabetes alone, in spite of the fact that strong cross-reactive responses with GAD67 are observed only in patients with APECED. The high GAD67Ab titres may indicate that sensitization to GAD67 occurs independently of GAD65 in patients with APECED, possibly reflecting a different pathogenic mechanism, as proposed by Björk et al. (19). This is supported by the fact that HLA-conferred susceptibility to type 1 diabetes has not been observed in patients with APECED (8). The isotype pattern of GAD65 antibodies in young children at risk of developing type 1 diabetes comprises a dominant IgG1 response, more infrequent IgG2 and IgG4 responses and rarely an IgG3 response $(18,22)$, although the last-mentioned subclass response may be somewhat more frequent in the initial phase (M Ronkainen, unpublished data). In the present series, a similar pattern apart from the absence of an IgG3 response was observed in the samples taken at the time of diagnosis from the patients with type 1 diabetes alone. The isotype profile seen in APECED patients, irrespective of the presence of concomitant type 1 diabetes, was very similar to that of the patients with type 1 diabetes alone but only the latter had IgG2 antibodies frequently. An IgG3 response was seen initially in only two non-diabetic APECED patients, one of whom was one of the youngest patients in the series. This is also consistent with our previous observation of the occurrence of IgG3 subclass antibodies during the initial GAD response. The apparently increased frequency of IgG4 responses among the non-diabetic APECED patients relative to the diabetic ones $(79 \%$ vs $50 \%)$ could be interpreted as a sign of non-destructive autoimmunity. This would be in line with our observations in a prospective study of young GAD65Ab-positive children that those who remained non-diabetic were characterized by an emerging IgG4 response over the first 18 months after seroconversion to GAD65Ab positivity (M Ronkainen et al., unpublished observations). Previously reported findings are not unequivocal in this respect, however $(18,22,25-27)$.

In conclusion, GAD65Abs in APECED patients target the middle and C-terminal regions and occasionally also the N-terminal region and comprise mainly the IgG1 subclass, although IgG2 and IgG4 subclass antibodies are also observed relatively frequently. The profile of their humoral immune response to GAD65 does not differ markedly from that of patients with type 1 diabetes alone, as only GAD65Abs of the IgG2 subclass are associated more closely with isolated type 1 diabetes. The antibody response to GAD is similar in diabetic APECED patients and in patients with type 1 diabetes alone. Our findings imply that it is not possible to discriminate APECED patients affected by type 1 diabetes from unaffected ones by means of epitope- and isotype-specific GAD antibodies. A strong humoral immune response to GAD67 characterizes APECEDrelated GAD autoimmunity and differentiates patients with type 1 diabetes alone from APECED patients, the former having, in addition, an increased IgG2 subclass response to GAD65.

\section{Acknowledgements}

We are grateful to Elina Ronkainen, Leni Joutsjoki and Jukka Rinkinen for their skilful assistance in the analyses of the inhibition samples. This work was supported by the Type 1 Diabetes Targeted Programme co-funded by the Research Council for Health, Academy of Finland, the Juvenile Diabetes Foundation International and the Sigrid Jusélius Foundation, the Medical Research Funds of Helsinki University Hospital and the Novo Nordisk Foundation.

\section{References}

1 Leslie RDG, Atkinson MA \& Notkins AL. Autoantigens IA-2 and GAD in type I (insulin-dependent) diabetes. Diabetologia 1999 $423-14$.

2 Velloso LA, Winqvist O, Gustafsson J, Kämpe O \& Karlsson EA. Autoantibodies against a novel $51 \mathrm{kDa}$ islet antigen and glutamate decarboxylase isoforms in autoimmune polyendocrine syndrome type I. Diabetologia 199437 61-69. 
3 Christie MR, Genovese S, Cassidy D, Bosi E, Brown TJ, Lai M, Bonifacio E \& Bottazzo G. Antibodies to islet $37 \mathrm{k}$ antigen, but not to glutamate decarboxylase, discriminate rapid progression to IDDM in endocrine autoimmunity. Diabetes $1994 \mathbf{4 3}$ $1254-1259$

4 Vianello M, Tavolato B \& Giometto B. Glutamic acid decarboxylase autoantibodies and neurological disorders. Neurological Sciences 200223 145-151.

5 Karlsen AE, Hagopian WA, Grubin CE, Dube S, Disteche CM, Adler DA, Bärmeier $\mathrm{H}$, Mathews S, Grant FJ, Foster D \& Lernmark $\AA$. Cloning and primary structure of a human islet isoform of glutamic acid decarboxylase from chromosome 10. PNAS 199188 8337-8341.

$6 \mathrm{Bu}$ DF, Erlander MG, Hitz BC, Tillakaratne NJK, Kaufman DL, Wagner-McPherson B, Evans GA \& Tobin AJ. Two human glutamate decarboxylases, 65-kDa GAD and 67-kDa GAD, are each encoded by a single gene. PNAS 199289 2115-2119.

7 Ujihara N, Daw K, Gianani R, Boel E, Yu L \& Powers AC. Identification of glutamic acid decarboxylase autoantibody heterogeneity and epitope regions in type 1 diabetes. Diabetes $199443968-975$.

8 Tuomi T, Björses P, Falorni A, Partanen J, Perheentupa J, Lernmark $\AA$ \& Miettinen A. Antibodies to glutamic acid decarboxylase and insulin-dependent diabetes in patients with autoimmune polyendocrine syndrome type I. Journal of Clinical Endocrinology and Metabolism 199681 1488-1494.

9 Björses P, Aaltonen J, Horelli-Kuitunen N, Yaspo ML \& Peltonen L. Gene defect behind APECED: a new clue to autoimmunity. Human Molecular Genetics 19987 1547-1553.

10 Ahonen P, Myllärniemi S, Sipilä I \& Perheentupa J. Clinical variation of autoimmune polyendocrinopathy-candidas-ectodermal dystrophy (APECED) in a series of 68 patients. New England Journal of Medicine 1990322 1829-1836.

11 Perheentupa J. APS-1/APECED: the clinical disease and therapy. Endocrinology and Metabolism Clinics of North America 200231 295-320.

12 Gylling M, Tuomi T, Björses P, Kontiainen S, Partanen J, Christie MR, Knip M, Perheentupa J \& Miettinen A. $\beta$-Cell autoantibodies, human leukocyte antigen II alleles, and type 1 diabetes in autoimmune polyendocrinopathy-candidiasis-ectodermal dystrophy. Journal of Clinical Endocrinology and Metabolism $2000854434-4440$.

13 Kulmala P, Savola K, Petersen JS, Vähäsalo P, Karjalainen J, Löppönen T, Dyrberg T, Åkerblom HK Knip M \& the Childhood Diabetes in Finland Study Group. Prediction of insulin-dependent diabetes mellitus in siblings of children with diabetes - a population based study. Journal of Clinical Investigation $1998101327-336$.

14 Richter W, Shi Y \& Baekkeskov S. Autoreactive epitopes defined by diabetes-associated human monoclonal antibodies are localized in the middle and C-terminal domains of the smaller form of glutamate decarboxylase. PNAS 199390 2832-2836.

15 Falorni A, Ackefors M, Carlberg C, Daniels T, Persson B, Robertson J \& Lernmark $\AA$. Diagnostic sensitivity of immunodominant epitopes of glutamic acid decarboxylase (GAD65) autoantibodies in childhood IDDM. Diabetologia $1996391091-1098$.
16 Schwartz HL, Chandonia J-M, Kash SF, Kanaani J, Tunnell E, Domingo A, Cohen FE, Banga JP, Madec A-M, Richter W \& Baekkeskov S. High-resolution autoreactive epitope mapping and structural modeling of the $65 \mathrm{kDa}$ form of human glutamic acid decarboxylase. Journal of Molecular Biology 1999 287 983-999.

17 Bonifacio E, Lampasona V, Bernasconi I \& Ziegler AG. Maturation of the humoral response to epitopes of GAD in preclinical childhood type 1 diabetes. Diabetes $200049202-208$.

18 Bonifacio E, Scirpoli M, Kredel K, Füchtenbusch M \& Ziegler AG. Early autoantibody responses in prediabetes are IgG1 dominated and suggest antigen-specific regulation. Journal of Immunology $1999163525-532$.

19 Björk E, Velloso LA, Kämpe O \& Karlsson FA. GAD autoantibodies in IDDM, stiff-man syndrome, and autoimmune polyendocrine syndrome type I recognise different epitopes. Diabetes 199443 161-165.

20 Sabbah E, Savola K, Ebeling T, Kulmala P, Vähäsalo P, Salmela PI, Ilonen J \& Knip M. Genetic, autoimmune and clinical characteristics of childhood and adult onset type 1 diabetes. Diabetes Care $2000231326-1332$.

21 Savola K, Sabbah E, Kulmala P, Vähäsalo P, Ilonen J \& Knip M. Autoantibodies associated with type 1 diabetes mellitus persist after diagnosis in children. Diabetologia $1998 \mathbf{4 1} 1293-1297$.

22 Hoppu S, Ronkainen MS, Kulmala P, Åkerblom HK Knip M \& the Childhood Diabetes in Finland Study Group. GAD65 antibody isotypes and epitope recognition during the prediabetic process in siblings of children with type I diabetes. Clinical and Experimental Immunology 2004136 120-128.

23 Solimena M, Folli F, Aparisi R, Pozza G \& De Camilli P. Autoantibodies to GABA-ergic neurons and pancreatic beta cells in stiffman syndrome. New England Journal of Medicine 1990322 1555-1560.

24 Kim J, Namchuk M, Bugawan T, Fu Q, Jaffe M, Shi Y, Aanstoot HJ, Turck CW, Erlich H, Lennon V \& Baekkeskov S. Higher autoantibody levels and recognition of a linear $\mathrm{NH}_{2}$-terminal epitope in the autoantigen GAD65, distinguish stiff-man syndrome from insulin-dependent diabetes mellitus. Journal of Experimental Medicine $1994 \mathbf{1 8 0} 595-606$.

25 Couper JJ, Harrison LC, Aldis JJE, Colman PG, Honeyman MC \& Ferrante A. IgG subclass antibodies to glutamic acid decarboxylase and risk for progression to clinical insulin-dependent diabetes. Human Immunology 199859 493-499.

26 Petersen JS, Kulmala P, Clausen JT, Knip M Dyrberg T \& the Childhood Diabetes in Finland Study Group. Progression to type 1 diabetes is associated with a change in the immunoglobulin isotype profile of autoantibodies to glutamic acid decarboxylase (GAD65). Clinical Immunology 199990 276-281.

27 Hawa MI, Fava D, Medici F, Deng Y-J, Notkins AL, De Mattia G \& Leslie RDG. Antibodies to IA-2 and GAD65 in type 1 and type 2 diabetes. Diabetes Care 200023 228-233.

Received 27 April 2005

Accepted 1 September 2005 